\title{
OTIMIZAÇÃO DO PROCESSO DE SECAGEM DOS FRUTOS DO AÇAÍ (Euterpe oleracea Mart.) EM SECADOR DE LEITO FIXO.
}

\author{
A. G. P. SILVA ${ }^{1}$, L. J. G. FARIA ${ }^{1}$, C. M. L. $\operatorname{COSTA}^{1}$ \\ ${ }^{1}$ Universidade Federal do Pará, Faculdade de Engenharia Química \\ E-mail para contato: adriano.silva@ itec.ufpa.br
}

\begin{abstract}
RESUMO: Antocianinas são corantes naturais que conferem cor a folhas, flores e frutas, apresentam potencial para uso como corante, além de atividade antioxidante e terapêutica. Essas características estimulam buscar formas de utilização desses corantes na indústria de diversos segmentos, além de fomentar pesquisas acerca de sua estabilidade. Nesse trabalho, realizou-se o estudo da secagem dos frutos de açaí (Euterpe oleracea Mart.) em secador de leito fixo, avaliando estatisticamente as influências das variáveis de entrada temperatura $(T)$, tempo de secagem (t) e vazão do ar de secagem (Q) nas respostas: razão de umidade e degradação das antocianinas presente no fruto do açaí. Foram obtidas as curvas de cinética de secagem nas temperaturas de 50,60 e $70{ }^{\circ} \mathrm{C}$ e nas vazões de $0,120,0,323$ e $0,340 \mathrm{~m}^{3} / \mathrm{s}$. Dentre os modelos de cinética de secagem avaliados o modelo de Midilli et al. foi o que melhor descreveu os dados experimentais. A condição ótima de secagem foi avaliada utilizando o conceito de desejabilidade global. Dentre as condições estabelecidas neste trabalho, o valor ótimo da Função Desejabilidade é quando $\mathrm{T}$ é deslocada para ao nível mais alto $\left(70{ }^{\circ} \mathrm{C}\right), \mathrm{t}$ para o mínimo (120min) e Q para o nível máximo $\left(340 \mathrm{~m}^{3} / \mathrm{s}\right)$.
\end{abstract}

\section{INTRODUÇÃO}

Antocianinas são compostos com diversas aplicações industriais como corantes e podem ser obtidas a partir de fontes naturais acessíveis. Corantes são substancias que conferem, intensificam ou restauram a cor de um alimento e são classificados como aditivos alimentares. A utilização de corantes naturais que podem substituir corantes artificiais é relevante tendo em vista que os artificiais são potencialmente tóxicos e podem causar reações adversas nos consumidores.

O açaí, fruto tipicamente tropical, surge como uma promissora opção, uma vez que é encontrado em abundância no Norte do Brasil e apresenta alto teor de antocianinas. Além destes fatores, o açaí, possui um grande apelo mercadológico, sendo considerado um fruto muito nutritivo (ROGEZ, 2000). Para melhor aproveitar o potencial dos corantes naturais são imprescindíveis estudos na busca de soluções para questões como novas fontes e estabilidade. Assim, a obtenção de um produto final de fácil manuseio, armazenagem e principalmente de maior estabilidade, assume grande importância. 
A secagem é uma das principais operações de pré-processamento das fontes naturais, influenciando nas propriedades químicas e físicas dos compostos extraídos dessas fontes. Devido a complexidade de se trabalhar com produtos naturais, o processo de secagem e os requisitos necessários à armazenagem, devem ser realizados mediante métodos estatísticos apropriados, com os quais é possível analisar os efeitos das variáveis estudadas.

Este trabalho tem como principal objetivo otimizar a secagem dos frutos de açaí (Euterpe oleracea Mart.), com base na técnica de planejamento fatorial Box-Behnken, utilizando um secador de leito fixo. Os efeitos das variáveis de controle (temperatura, tempo e vazão do ar de secagem), na qualidade dos frutos foram avaliados através das variáveis de resposta: umidade final e degradação de antocianinas.

\section{MATERIAIS E MÉTODOS}

Os frutos do açaí foram adquiridos na Feira do Açaí em Belém-Pará nos meses de outubro e dezembro de 2013 e levados ao laboratório, os quais foram imediatamente congelados em freezer até a realização dos experimentos.

\subsection{Sistema Experimental}

A secagem foi realizada em um secador de leito fixo o qual consiste de um duto de $25,4 \mathrm{~cm}$ de seção quadrada, contendo um soprador centrífugo com controle de velocidade. Compõe ainda o secador dois pares de resistência elétricas de $2 \mathrm{~kW}$ e $1 \mathrm{~kW}$ cada, propiciando o aquecimento do ar. Adaptado a esta unidade, encontra-se o corpo do secador constituído de vidro pirex, contendo dispositivos para medidas de temperatura do ar. $\mathrm{O}$ equipamento dispõe ainda de um cesto cilíndrico telado, em aço inoxidável, no interior do qual são depositadas os frutos de açaí, ficando sujeitos ao contato do ar de secagem a partir de sua base e pelas laterais do dispositivo. Este cesto possui diâmetro interno $12,5 \mathrm{~cm}$ e altura $25,5 \mathrm{~cm}$. No decorrer do experimento, fica suspenso no interior do corpo cilíndrico de vidro, adaptado a uma balança eletrônica, que indica continuamente o decréscimo da massa do material.

\subsection{Ensaios de Cinética de Secagem}

Os ensaios de secagem foram conduzidos nas temperaturas de 50,60 e $70{ }^{\circ} \mathrm{C}$, nas vazões de $0,120,0,323$ e $0,340 \mathrm{~m}^{3} / \mathrm{s}$, mantendo constante a massa frutos de açaí previamente descongelados em $1500 \mathrm{~g}$. O cálculo da razão de umidade $(\mathrm{Xr})$ do produto, durante as diferentes condições experimentais, foi estimado pela Equação 1, sendo: $\mathrm{Xr}$ a razão de umidade, adimensional; $X_{t}$ é o teor de umidade no tempo t, decimal b.s e $X_{o}$ denota teor de umidade inicial do produto, decimal b.s.

$$
X r=\frac{X_{t}}{X_{o}}
$$

As massas de sólido seco, após os processos de secagem, necessárias ao cálculo dos teores de umidade em base seca, foram determinadas diretamente em estufa com circulação de ar forçada, a $105 \pm 1{ }^{\circ} \mathrm{C}$ por 24 horas, conforme metodologia padronizada. 
Os teores de umidade adimensionais em função do tempo de secagem foram avaliados por seis modelos matemáticos conforme sumarizados na Tabela 1 e descritas nos trabalhos de Radünz et al. (2010) e Meziane (2011). A escolha do modelo mais adequado foi realizada com base nas estatísticas: coeficiente de determinação $\left(\mathrm{R}^{2}\right)$, o erro médio relativo (EMR), dado pela equação 2, e pela análise dos gráficos da distribuição dos resíduos (aleatório ou tendencioso).

$$
D=\frac{100}{N} \sum \frac{\left|Y-Y^{\prime}\right|}{Y}
$$

Tabela 1 - Modelos matemáticos utilizados nos ajustes da cinética de secagem

\begin{tabular}{c|c}
\hline Modelo & Equação \\
\hline \hline Dois Termos & $X r=a \cdot e^{\left(-k_{0} t\right)}+b \cdot e^{\left(-k_{1} t\right)}$ \\
Henderson e Pabis modificado & $X r=a \cdot e^{(-k t)}+b \cdot e^{(-g t)}+c \cdot e^{(-h t)}$ \\
Midilliet al. & $X r=a \cdot e^{\left(-k t^{n}\right)}+b \cdot t$ \\
Page & $X_{R}=e^{\left(-K t^{n}\right)}$ \\
Page modificado & $X r=e^{(-k t)^{n}}$ \\
Verma & $X r=a \cdot e^{(-k t)}+(1-a) \cdot e^{(-g t)}$ \\
\hline
\end{tabular}

\subsection{Planejamento Estatístico}

Foram realizadas 15 corridas experimentais, sendo três no ponto central conforme o planejamento de Box-Behnken. As variáveis de entrada foram: Temperatura $(T)$, tempo $(\mathrm{t})$ e vazão do ar (Q); codificadas como $\mathrm{X}_{1}, \mathrm{X}_{2}$ e $\mathrm{X}_{3}$, respectivamente. As variáveis de resposta analisadas foram: Razão de umidade (Xr); adimensional e degradação das antocianinas (D); porcentagem. Na Tabela 2 são apresentados os valores das variáveis de entrada do processo.

Tabela 2 - Variáveis de entrada e seus respectivos níveis

\begin{tabular}{c|c|c|c|c}
\hline \multirow{2}{*}{ Variáveis } & \multirow{2}{*}{ Notação } & \multicolumn{3}{|c}{ Níveis } \\
\cline { 3 - 5 } & & -1 & 0 & 1 \\
\hline \hline $\mathrm{T}\left({ }^{\circ} \mathrm{C}\right)$ & $\mathrm{X}_{1}$ & 50 & 60 & 70 \\
$\mathrm{t}(\mathrm{min})$ & $\mathrm{X}_{2}$ & 120 & 240 & 360 \\
$\mathrm{Q}\left(\mathrm{m}^{3} / \mathrm{s}\right)$ & $\mathrm{X}_{3}$ & 0,120 & 0,323 & 0,340 \\
\hline
\end{tabular}

\subsection{Extração das antocianinas}

Após a secagem, de $30 \mathrm{~g}$ dos frutos foram utilizados para avaliar a percentagem de degradação das antocianinas. A análise quantitativa das antocianinas extraídas dos frutos do açaí foi realizada de acordo com o método de Fuleki e Francis (1968) posteriormente revisada por Lees e Francis (1972).O tempo e a forma de extração foram conduzidos conforme a metodologia de Constant,(2003). Utilizando pH único, onde após 24 h, as amostras foram filtradas, uma alíquota de $1 \mathrm{ml}$ de cada extrato foi diluída em $10 \mathrm{ml}$ de etanol $95 \% \mathrm{com} \mathrm{HCl}$ 1,5 N (85:15), deixada em repouso em ausência de luz por $2 \mathrm{~h}$ e levada à leitura espectrofotométrica da absorbância em $535 \mathrm{~nm}$. As análises de absorbância foram feitas em 
espectrofotômetro UV-Visível, realizadasem duplicata e para o cálculo da concentração utilizou-se a Equação 3, em termos de cianidina:

$\operatorname{Antocioninas}(m g \mid 100 g$ de amostra $)=\frac{A b s \lambda_{\max } \cdot F d .100}{E_{1 c m}^{1 \%}}(3)$

Em que: Abs $\lambda_{\text {máx }}$ é a absorbância no comprimento de onda máximo (535nm); Fd é o fator de diluição e $\mathrm{E}^{1 \%} \mathrm{~cm}$ (absortividade molar a 535nm) = 98,29 (antocianinas de Cranberries).

\section{RESULTADOS E DISCUSSÃO}

\subsection{Cinética de Secagem}

Na Tabela 3 são apresentados os valores dos parâmetros estatísticos(coeficiente de determinação - $\mathrm{R}^{2}$, erro médio relativo - EMR, e análise dos gráficos de resíduos - $\mathrm{R}$ ) utilizados para avaliar os modelos matemáticos que descrevem a cinética de secagem dos frutos de açaí em leito fixo.

Tabela 3 -Avaliação da qualidade dos modelos matemáticosutilizados na cinética de secagem

\begin{tabular}{c|c|ccc|ccc|ccc}
\hline \multirow{2}{*}{ Modelo } & \multirow{2}{*}{$\mathrm{T}\left({ }^{\circ} \mathrm{C}\right)$} & \multicolumn{3}{|c|}{$\mathrm{Q}=0,340 \mathrm{~m}^{3} / \mathrm{s}$} & \multicolumn{3}{c|}{$\mathrm{Q}=0,323 \mathrm{~m}^{3} / \mathrm{s}$} & \multicolumn{3}{c}{$\mathrm{Q}=0,120 \mathrm{~m}^{3} / \mathrm{s}$} \\
\cline { 3 - 10 } & & $\mathrm{R}^{2}(\%)$ & $\mathrm{EMR}(\%)$ & $\mathrm{R}$ & $\mathrm{R}^{2}(\%)$ & $\mathrm{EMR}(\%)$ & $\mathrm{R}$ & $\mathrm{R}^{2}(\%)$ & $\mathrm{EMR}(\%)$ & $\mathrm{R}$ \\
\hline \hline \multirow{3}{*}{ Dois Termos } & 50 & 99,89 & 0,096 & $\mathrm{~A}$ & 98,1 & 0,293 & $\mathrm{~T}$ & 99,58 & 0,131 & $\mathrm{~A}$ \\
& 60 & 99,37 & 0,408 & $\mathrm{~A}$ & 98,71 & 0,327 & $\mathrm{~T}$ & 96,72 & 0,512 & $\mathrm{~A}$ \\
& 70 & 98,72 & 0,669 & $\mathrm{~T}$ & 99,6 & 0,348 & $\mathrm{~T}$ & 97,83 & 0,561 & $\mathrm{~A}$ \\
\hline \multirow{2}{*}{ Henderson e Pabis } & 50 & 97,55 & 0,438 & $\mathrm{~T}$ & 98,12 & 0,302 & $\mathrm{~T}$ & 99,58 & 0,131 & $\mathrm{~A}$ \\
modificado & 60 & 99,08 & 0,464 & $\mathrm{~A}$ & 98,72 & 0,326 & $\mathrm{~T}$ & 96,76 & 0,512 & $\mathrm{~A}$ \\
& 70 & 98,72 & 0,669 & $\mathrm{~A}$ & 99,63 & 0,325 & $\mathrm{~T}$ & 97,83 & 0,561 & $\mathrm{~A}$ \\
\hline \multirow{3}{*}{ Midilliet al. } & 50 & 99,95 & 0,068 & $\mathrm{~A}$ & 99,8 & 0,147 & $\mathrm{~A}$ & 99,65 & 0,113 & $\mathrm{~A}$ \\
& 60 & 99,57 & 0,348 & $\mathrm{~A}$ & 99,8 & 0,163 & $\mathrm{~A}$ & 99,15 & 0,262 & $\mathrm{~A}$ \\
& 70 & 99,09 & 0,617 & $\mathrm{~A}$ & 99,83 & 0,3 & $\mathrm{~A}$ & 99,68 & 0,2 & $\mathrm{~A}$ \\
\hline \multirow{3}{*}{ Page } & 50 & 98,25 & 0,408 & $\mathrm{~T}$ & 98,62 & 0,325 & $\mathrm{~T}$ & 99,49 & 0,147 & $\mathrm{~A}$ \\
& 60 & 99,19 & 0,368 & $\mathrm{~A}$ & 99,01 & 0,331 & $\mathrm{~A}$ & 97,9 & 0,124 & $\mathrm{~A}$ \\
\cline { 3 - 10 } Page modificado & 70 & 98,21 & 0,763 & $\mathrm{~A}$ & 99,42 & 0,431 & $\mathrm{~T}$ & 95,87 & 0,744 & $\mathrm{~A}$ \\
& 50 & 58,02 & 2,193 & $\mathrm{~T}$ & 85,28 & 1,277 & $\mathrm{~T}$ & 99,33 & 0,175 & $\mathrm{~T}$ \\
& 60 & 84,63 & 2,241 & $\mathrm{~T}$ & 89,57 & 1,355 & $\mathrm{~T}$ & 83,44 & 1,162 & $\mathrm{~T}$ \\
& 70 & 96,44 & 1,121 & $\mathrm{~T}$ & 98,97 & 0,596 & $\mathrm{~T}$ & 93,98 & 0,991 & $\mathrm{~A}$ \\
\hline \multirow{3}{*}{ Verma } & 50 & 99,89 & 0,096 & $\mathrm{~A}$ & 99,83 & 0,136 & $\mathrm{~A}$ & 99,63 & 0,118 & $\mathrm{~A}$ \\
& 60 & 99,37 & 0,407 & $\mathrm{~A}$ & 89,57 & 1,355 & $\mathrm{~T}$ & 99,13 & 0,272 & $\mathrm{~A}$ \\
& 70 & 99,24 & 0,611 & $\mathrm{~A}$ & 99,79 & 0,272 & $\mathrm{~A}$ & 99,75 & 0,168 & $\mathrm{~A}$ \\
\hline
\end{tabular}

A partir da análise da Tabela 2 observa-se que o modelo matemático de Midilli et al.(2002) foi o que melhor descreveu os dados da cinética de secagem para os três níveis de vazão do ar de secagem e na faixa de temperatura estudada, pois os coeficientes de determinação estão acima de $99 \%$, os valores para o erro médio relativo foram menores que 1 \% e a distribuição de resíduos foi totalmente aleatória. 
Com base no estudo da cinética de secagem dos frutos de açaí em leito fixo constatou-se que o equilíbrio termodinâmico entre o material e o ar atmosférico não foi alcançado dentro das condições experimentais avaliadas, porém a umidade diminui continuamente com o tempo de secagem e com o aumento da temperatura do ar, concordando assim com os fundamentos da teoria de secagem (Strumillo e Kudra, 1986).

Outro fator observado foi o efeito significativo da temperatura sobre as curvas de secagem, ou seja, com o aumento da temperatura ocorre maior taxa de remoção de água do material, fato encontrado por diversos pesquisadores para a secagem de biomateriais (Radünz et al., 2010; Kadam et al., 2011).

\subsection{Análise Estatística}

A Tabela 4 corresponde a matriz de experimentos com as variáveis de entrada originais e os resultados médios das variáveis de resposta. As corridas foram realizadas de forma randomizada para minimizar os erros experimentais.

Tabela 3 - Matriz de experimentos Box-Behnken e os resultados das variáveis de resposta.

\begin{tabular}{c|ccc|ccc|cc}
\hline \multirow{2}{*}{ Corrida } & \multicolumn{2}{|c|}{ Variáveis Codificadas } & \multicolumn{3}{|c|}{$\begin{array}{c}\text { Variáveis Originais } \\
\text { (unidade) }\end{array}$} & \multicolumn{2}{c}{$\begin{array}{c}\text { Variáveis de Resposta } \\
\text { (unidade) }\end{array}$} \\
\cline { 2 - 8 } & $\mathrm{X}_{1}$ & $\mathrm{X}_{2}$ & $\mathrm{X}_{3}$ & $\begin{array}{c}\mathrm{T} \\
\left({ }^{\mathrm{O}} \mathrm{C}\right)\end{array}$ & $\begin{array}{c}\mathrm{t} \\
(\mathrm{min})\end{array}$ & $\begin{array}{c}\mathrm{Q} \\
\left(\mathrm{m}^{3} / \mathrm{s}\right)\end{array}$ & $\begin{array}{c}\mathrm{X}_{\mathrm{R}} \\
(\text { adim. })\end{array}$ & $\begin{array}{c}\mathrm{D} \\
(\%)\end{array}$ \\
\hline \hline 1 & -1 & -1 & 0 & 50 & 120 & 0,323 & 0,86 & 42,29 \\
2 & 1 & -1 & 0 & 70 & 120 & 0,323 & 0,73 & 35,02 \\
3 & -1 & 1 & 0 & 50 & 360 & 0,323 & 0,70 & 70,35 \\
4 & 1 & 1 & 0 & 70 & 360 & 0,323 & 0,53 & 46,71 \\
5 & -1 & 0 & -1 & 50 & 240 & 0,120 & 0,83 & 59,19 \\
6 & 1 & 0 & -1 & 70 & 240 & 0,120 & 0,74 & 52,69 \\
7 & -1 & 0 & 1 & 50 & 240 & 0,340 & 0,54 & 76,47 \\
8 & 1 & 0 & 1 & 70 & 240 & 0,340 & 0,56 & 71,9 \\
9 & 0 & -1 & -1 & 60 & 120 & 0,120 & 0,87 & 20,96 \\
10 & 0 & 1 & -1 & 60 & 360 & 0,120 & 0,64 & 78,66 \\
11 & 0 & -1 & 1 & 60 & 120 & 0,340 & 0,78 & 42,41 \\
12 & 0 & 1 & 1 & 60 & 360 & 0,340 & 0,56 & 80,69 \\
13 & 0 & 0 & 0 & 60 & 240 & 0,323 & 0,73 & 73,93 \\
14 & 0 & 0 & 0 & 60 & 240 & 0,323 & 0,72 & 60,84 \\
15 & 0 & 0 & 0 & 60 & 240 & 0,323 & 0,70 & 67,56 \\
\hline
\end{tabular}

Contrário ao que se imaginava, a análise da Tabela 3 demonstrou que as maiores degradações de antocianinas ocorreram nas corridas realizadas com as menores temperaturas, quando apenas a temperatura de secagem foi o parâmetro diferencial. Este fato pode ser verificado na corrida $2\left(70{ }^{\circ} \mathrm{C}\right)$ a qual gerou menor degradação de antocianinas que a corrida 1 $\left(50{ }^{\circ} \mathrm{C}\right)$. Este fato também pode ser verificado quando se comparou as corridas 3-4, 5-6 e 7-8.

Segundo Gonnet (1998) as antocianinas são estáveis e se encontram na forma colorida como cátion flavilium apenas em soluções bastante ácidas. Lewis et al. (1995) afirma que a proteção do cátion flavilium contra a hidratação é absolutamente necessária para manter a coloração das soluções, ou seja, uma maneira de reter esta coloração seria pela remoção da 
água do meio o que promoveria o deslocamento do equilíbrio hidratação/desidratação para direção da forma colorida $\mathrm{AH}^{+}$. Logo, essa seria uma possível explicação para o fato de ter se encontrado valores mais baixos de degradação de antocianinas nas secagens realizadas com as maiores temperaturas,poisessa condição experimental (temperatura mais alta) favorece a remoção umidade dos frutos de açaí, proporcionado assim a forma colorida $\mathrm{AH}^{+}$, coloração esta que é indicadora da estabilidade das antocianinas.

Visando analisar os efeitos das variáveis de controle nas respostas realizou-se a análise da ANOVA para as respostas $\mathrm{Xr}$ e D (Tabela 5) onde foram considerados os níveis de significância $\mathrm{p}=0,01$ (confiança de $99 \%$ ), $\mathrm{p}=0,05$ (confiança de $95 \%$ ), e p=0,10 (confiança de $90 \%$ ). A análise da ANOVA demonstra que apenas as variáveis de controle isoladas $\mathrm{X}_{1}$, $\mathrm{X}_{2}$ e $\mathrm{X}_{3}$ influenciam na variável de resposta $\mathrm{Xr}$ nos limites de rejeição estabelecidos, por apresentar uma probabilidade pequena de estar dentro da região de hipótese nula (estatística $\mathrm{P})$ e por apresentar valores de $\mathrm{F}_{\text {calculado }}$ maior que o do $\mathrm{F}_{\text {tabelado }}\left(\mathrm{F}_{0,01 ; 1 ; 5}=16,26\right.$ para $99 \%$ de confiança; $\mathrm{F}_{0,05 ; 1 ; 5}=6,61$ para $95 \%$ de confiança e $\mathrm{F}_{0,1 ; 1 ; 5}=4,06$ para $90 \%$ de confiança).

Tabela 5 - Análise de variância ANOVA para as variáveis de resposta Xr e D

\begin{tabular}{c|cc|cc|cc|cc|cc}
\hline \multirow{2}{*}{ Efeitos } & \multicolumn{2}{|c|}{$\begin{array}{c}\text { Soma dos } \\
\text { Quadrados }\end{array}$} & \multicolumn{2}{c|}{$\begin{array}{c}\text { Graus de } \\
\text { liberdade }\end{array}$} & \multicolumn{2}{c|}{$\begin{array}{c}\text { Quadrado } \\
\text { Médio }\end{array}$} & \multicolumn{2}{c|}{ Teste F } & \multicolumn{2}{|c}{ Probabilidade (P) } \\
\cline { 2 - 12 } & $\mathrm{Xr}$ & $\mathrm{D}$ & $\mathrm{Xr}$ & $\mathrm{D}$ & $\mathrm{Xr}$ & $\mathrm{D}$ & $\mathrm{Xr}$ & $\mathrm{D}$ & $\mathrm{Xr}$ & $\mathrm{D}$ \\
\hline \hline $\mathrm{X}_{1}$ & 0,0183 & 220,28 & 1 & 1 & 0,018 & 220,28 & 4,78 & 1,99 & $0,0804^{* * *}$ & 0,2167 \\
$\mathrm{X}_{2}$ & 0,0846 & 2303,05 & 1 & 1 & 0,084 & 2303,05 & 22,15 & 20,89 & $0,0053^{*}$ & $0,0060^{*}$ \\
$\mathrm{X}_{3}$ & 0,0504 & 449,48 & 1 & 1 & 0,050 & 449,48 & 13,20 & 4,08 & $0,0150^{* *}$ & $0,0995^{* * *}$ \\
$\mathrm{X}_{1}{ }^{2}$ & 0,0035 & 82,71 & 1 & 1 & 0,003 & 82,71 & 0,91 & 0,75 & 0,3836 & 0,4261 \\
$\mathrm{X}_{2}{ }^{2}$ & 0,0010 & 735,80 & 1 & 1 & 0,0010 & 735,80 & 0,25 & 6,67 & 0,6344 & $0,0492^{* *}$ \\
$\mathrm{X}_{3}{ }^{2}$ & 0,0017 & 20,47 & 1 & 1 & 0,0017 & 20,47 & 0,45 & 0,18 & 0,5336 & 0,6845 \\
$\mathrm{X}_{1} \mathrm{X}_{2}$ & 0,0003 & 67 & 1 & 1 & 0,0003 & 67,00 & 0,07 & 0,61 & 0,7971 & 0,4709 \\
$\mathrm{X}_{1} \mathrm{X}_{3}$ & 0,0033 & 0,926 & 1 & 1 & 0,0033 & 0,93 & 0,85 & 0,01 & 0,3977 & 0,9305 \\
$\mathrm{X}_{2} \mathrm{X}_{3}$ & 0,000012 & 94,369 & 1 & 1 & $1,2 \mathrm{E}-05$ & 94,37 & 0,003 & 0,85 & 0,9567 & 0,3974 \\
Erro & 0,0191 & 551,31 & 5 & 5 & 0,0038 & 110,26 & - & - & - & - \\
Total & 0,1822 & 4522,78 & 14 & 14 & - & - & - & - & - & - \\
\hline
\end{tabular}

*Significativo a $1 \% * *$ Significativo a $5 \% * * *$ Significativo a $10 \%$

Já para a variável $D$, verifica-se que o valor de $P$ para as variáveis $X_{2}, X_{3} e X_{2}^{2}$ se encontra fora dos limites de probabilidade estabelecidos para a hipótese nula e a análise do teste $\mathrm{F}$ confirma que essas variáveis são significativas para resposta avaliada (D), pois os valores de $F_{\text {calculado }}$ são maiores que os valores de $F_{\text {tabelado }}\left(F_{0,01 ; 1 ; 5}=16,26\right.$ para $99 \%$ de confiança; $\mathrm{F}_{0,05 ; 1 ; 5}=6,61$ para $95 \%$ de confiança e $\mathrm{F}_{0,1 ; 1 ; 5}=4,06$ para $90 \%$ de confiança).

\subsection{Otimização das Variáveis Independentes}

Para a otimização estatística do processo de secagem são atribuídos o valor 1 (valor desejado) para os limites inferiores e 0 (valor indesejado) para os limites medianos e superiores nas resposta $\mathrm{Xr}$ e D. Para os valores dos expoentes $s$ e $t$, que servem como ajuste para se encontrar o valor médio desejado da função foi atribuído o valor 20 para ambas as respostas. 
A análise do digrama da Função Desejabilidade (Figura 1) apresenta, dentro do domínio experimental, um valor otimizado em 0,8454 , que de acordo com a classificação de Akhanazarova e Kafarov (1982), considera-se essa resposta como aceitável e excelente.

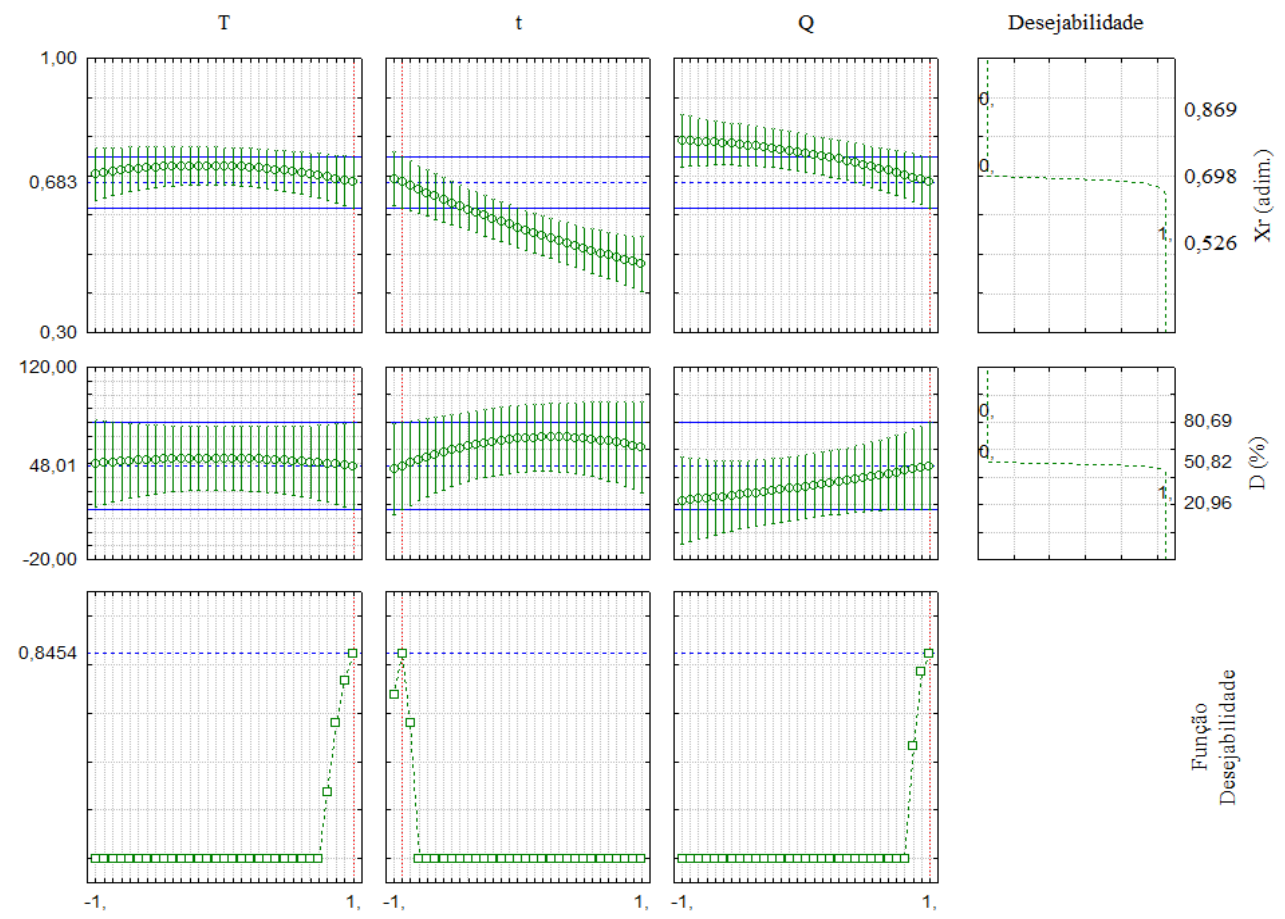

Figura 1 - Perfis para os valores preditos e a função desejabilidade

As condições estabelecidas como ótimas para as variáveis de resposta $\mathrm{Xr}$ e $\mathrm{D}$ correspondem a 0,683 e 48,01, respectivamente. Estes valores são alcançados para a combinação dos níveis máximo (+1), mínimo (-1) e máximo (+1) das variáveis independentes: temperatura do ar de secagem, tempo de secagem e vazão do ar de secagem, respectivamente. Dessa forma, o valor ótimo da função desejabilidade ocorre quando $\mathrm{T}$ é deslocada para o nível mais alto $\left(70{ }^{\circ} \mathrm{C}\right)$, t para o nível mínimo 120 (min) e Q para o nível máximo $\left(340 \mathrm{~m}^{3} / \mathrm{s}\right)$.

A condição ótima estabelecida para a variável $\mathrm{Xr}$ corresponde a uma razão final de umidade no valor de $0,683\left(\mathrm{X}_{\mathrm{f}}=49 \% \mathrm{bs}\right)$, o que ainda é considerado um valor muito acima daquele recomendado (em torno de $10 \%$ ) para a conservação de produtos naturais caso necessitem ser conservados nas condições ambientais de estocagem. Valores de umidade acima do recomendado elevam consideravelmente a possiblidade de deterioração dos produtos naturais por microrganismos, alterando assim suas características químicas e físicas.

Quanto ao valor da condição ótima estabelecida para a variável $\mathrm{D}$, verifica-se que este representa 48,01 \% da quantidade inicial de antocianinas, ou seja, ajustando-se as condições operacionais ótimas da secagem em leito fixo o teor de antocianinas diminui quase que pela metade do valor inicial de antocianina após da secagem.

Portanto verifica-se que dentro das condições experimentais adotadas neste estudo, o secador de leito fixo não foi capaz de reduzir, para níveis adequados, o teor de umidade e ainda reduziu para cerca de $50 \%$ o teor inicial de antocianinas presentes nos frutos do açaí. 


\section{CONCLUSÕES}

- Foi possível descrever o comportamento da cinética de secagem dos frutos de açaí a partir do modelo matemático de Midilli et al.(2002) para as condições experimentais estudada.

- As variáveis de controle isoladas $\mathrm{T}\left({ }^{\circ} \mathrm{C}\right), \mathrm{t}(\mathrm{min})$ e $\mathrm{Q}\left(\mathrm{m}^{3} / \mathrm{s}\right)$ influenciaram significativamente a variável de resposta razão de umidade $(\mathrm{Xr})$ nos limites de rejeição estabelecidos.

- As variáveis $t$ (min)em suas formas linear e quadrática e Q $\left(\mathrm{m}^{3} / \mathrm{s}\right)$ influenciaram significativamente a variável de resposta degradação de antocianinas (D).

- Dentro das condições experimentais adotadas neste estudo, o secador de leito fixo não foi capaz de reduzir, para níveis adequados, a razão de umidade ótima já que esta foi de 0,683 (aproximadamente $48 \%$ b.s) e ainda reduziu para cerca de $50 \%$ o teor inicial de antocioninas presentes nos frutos do açaí.

\section{REFERENCIAS}

AKHNAZAROVA, S.; KAFAROV, V. Experiment optimization in chemistry and chemical engineering. Moscow: Mir, 1982.

CONSTANT, P. B. L. Extração, caracterização e aplicação e antocianinas e açaí. 2003. 199 f. Tese (Doutorado em Ciência e Tecnologia de Alimentos) - UFV, MG, 2003.

FULEKI, T. e FRANCIS, F. J. Quantitative methods for anthocyanins.1 Extraction and determination of total anthocyanin in craberries. Jounal of Food Science. v. 33, p.72-77, 1968.

GONNET, J. F. Colour effects of co-pigmentation of anthocyanins revisited-1. A colorimetric definition using the CIELAB scale. Food Chemistry, v.63, n.3, p.409-415, 1998

KADAM, D. M.; GOYAL, R. K.; SINGH, K. K.; GUPTA, M. K. Thin layer convective drying of mint leaves. J. Med. Plants Res, v. 5, p. 164-170, 2011

LEES, D. H.; FRANCIS, F. G. Standardization of pigment analysis in cramberries. Hortscience, [S. 1.], v. 7, p. 83-84, 1972.

LEWIS, C. E., WALKER, J. R. L., LANCASTER, J. E. Effect of polysaccharides on the colour of anthocyanins. Food Chemistry. v. 45, p. 315-319, 1995.

MEZIANE, S. Drying kinetics of olive pomace in a fluidized bed dryer. Energy Conversionand Management. v. 52, p. 1644-1649, 2011

MIDILLI, A.; KUCUK, H.; YAPAR, Z.A new model for single-layer drying. Drying Technology, Philadelphia, v.20, n.7, p.1503-1513, 2002.

RADÜNZ, L. L.; MOSSI, A. J.; ZAKRZEVSKI, C. A.; AMARAL, A. S. GRASSMANN, L. Análise da cinética de secagem de folhas de sálvia. Rev. Bras. Eng. Agríc. Ambient, v. 14, p. 979-986, 2010.

ROGEZ, H. Açaí: Preparo, composição e melhoramento da conservação. Belém: EdUFPA, 313 p. 2000.

STRUMILLO, C.; KUDRA, T. Drying: principles, applications and design. New York: Gordon and Breach Science Publishers, (Topics in chemical engineering, v. 3), 1986. 